\title{
IMMUNOHISTOCHEMICAL LOCALIZATION OF CONNEXIN 43 IN THE CORNEA OF THE ONE HUMPED CAMEL (CAMELUS DROMEDARIES)
}

\author{
ENAS A.A. ELHAFEZ ${ }^{*}$ and KHALED ALY* \\ *Department of Anatomy and Histology, Faculty of Veterinary Medicine, Assiut University, Assiut 7562, Egypt. \\ Email: N/A
}

\section{ABSTRACT}

Received at: $20 / 12 / 2014$

Accepted: 16/2/2015
The present study has described for the first time the expression of connexin 43 in the cornea of camel. The camel cornea was composed of five layers, the corneal epithelium, subepithelial basement membrane (Bowman's membrane), substantiapropria or stroma, posterior limiting membrane (Descemet's membrane), and posterior epithelium (corneal endothelium). Using immunohistochemical techniques we have examined the localizations of the connexin 43 in one humped camel cornea. The expression of gap junction protein CX43 was appeared as punctate staining pattern along the lateral plasma membrane between the cells and at the apical surface. We observed the expression in both basal and suprabasal cell layers; the superficial cell layer was give negative staining reactions for CX 43 . While the fibroblast cells of the corneal stroma revealed moderate immuno-staining for connexin 43. However no reaction was evident in the collagen fiber of the stroma.

Keywords: Connexin 43; cornea; camel; immunohistochemistry.

\section{INTRODUCTION}

Gap Junctions are intercellular channels that allow low moleculuar weight substances to pass between cells. These junctions are formed by connexins (CX) that are encoded by a multi gene family of more than 20 members. Protein structure analysis suggest that connexins share a common sequence of structural motifis, including 4 hydrophobic transmembrane, 2 extracellular domains, and 3 cytoplasmic domains (Yeager et al., 1996). Six connexins molecules assemble to form a ring structure known as connexin. (CX43) is the most widely expressed connexin, and its expression is also found in corneal epithelium (Cao et al., 1998). Gap junctions are cell-cell communicating channels that consist of multimeric proteins called connexins and mediate the change of low molecular weight metabolites and ions between contacting cells (Hertzberg et al., 2000 and Saez et al., 2003). Among their products, connexin43 (CX43) is the most widely expressed connexin and its expression is also found in the corneal endothelium (Mohay et al., 1995, Joyce et al., 1998, Williams et al., 2002, Laux et al., 2003 and Williams et al., 2004).

\section{MATERIALS and METHODS}

\section{Examined materials}

Eyeballs were obtained from 5 adult clinically healthy camels of both sexes.
These materials were collected from the Cairo and Assiut slaughterhouse.

\section{Reagents}

Anti-Cx43 primary antibody was obtained from Zytomed (Berlin, Germany). Mouse anti-rabbit and rabbit anti-mouse immunoglobulins and mouse alkaline phosphatase anti-alkaline phosphatase (APAAP) antibody complex were purchased from DAKO (Carpinteria, CA, USA). Immunoreaction visualization kit was from HistoMark (KLP, USA) and primers were obtained from MWG (Ebersberg, Germany).

\section{Light microscope}

The eyeballs were isolated from the head and then dissected by a sharp scalpel into two halves. Specimens were taken from the cornea, with a side length of $0.3-0.5 \mathrm{~cm}$. The samples were fixed in Bouin's solution and in 7\% buffered formalin solution. Fixation took 24 hours. They were then preserved in $70 \%$ ethanol for 24 hours until embedding (Bancroft 2002). After fixation the specimens were dehydrated in a graded series of alcohol then embedded. Finally the prepared tissue specimens were embedded in paraplast blocks. Sections were cut using a Leitz rotatory microtome (type 1516) at $4-5 \mu \mathrm{m}$.

\section{Histological Staining and examination}

Sections were deparafinized in xylene, then rehyderated in ethanol of descending concentration down to distilled water. After staining they were 
dehydrated in ascending concentrations of ethanol, clarified in xylene and covered with Canada Balsam. Different staining procedures were performed, Haematoxylin and Eosin staining (H\&E) for detection the general histological structure of the examined camel cornea. Periodic Acid Schiff reaction (PAS) for detection of the mucopolysaccharides and Trichrom staining for detection of connective tissue according to (Bancroft 2002). The stained sections were examined using LeitzDialux 20 Microscope. Photos were taken by using Agfa Pan 25 ASA, Kodak, Tri-X pan, 400ASA, Kodak, or Ectachrome plastic, 64 ASA films.

\section{Immunohistochemistry}

Immunohistochemical staining for $\mathrm{Cx} 43$ on consecutive paraffin sections was carried. Briefly, sections in sodium citrate buffer $(\mathrm{pH}$ 6.0), were incubated in a microwave at 1000 watts for 30 minutes. Endogenous peroxidases were inactivated by incubation of sections with $0.3 \%$ hydrogen peroxide $\left(\mathrm{H}_{2} \mathrm{O}_{2}\right)$ in methanol for 20 minutes. Non-specific reactions were blocked by treatment with $5 \%$ bovine serum albumin in phosphate buffered saline containing $10 \%$ fetal calf serum for at least 30 minutes at room temperature. Sections were incubated with the polyclonal anti-Cx43 primary antibody (1:250) over night at $4{ }^{\circ} \mathrm{C}$. Sections were incubated with mouse anti-rabbit immunoglobulin $(1: 50)$ as a secondary antibody, with rabbit-antimouse immunoglobulin (1:50) as a third antibody and then with mouse alkaline phosphatase anti-alkaline phosphatase (APAAP) antibody complex (1:100) for 30 minutes each. The immunoreactions were visualized using Histo Mark kit. For each immunoreaction, control incubations were performed by substituting buffer for the primary antibody. The experiment was repeated at least twice.

\section{RESULTS}

\section{Light microscopic examination}

As in other species the camel cornea is composed of five layers, the corneal epithelium, subepithelial basement membrane (Bowman's membrane), substantiapropria or stroma, posterior limiting membrane (Descemet's membrane), and posterior epithelium (corneal endothelium) (Fig. A). The outermost layer, the corneal epithelium is a stratified squamous epithelium. It consists of 14-17 layers of epithelial cells. The basal cell layer of corneal epithelium is represented by columnar cells resting on a wavy, thin and distinct basement membrane that stain positively with PAS (Fig. C) The height of the basal cell layer is variable depending upon the wavy character of the basement membrane, being relatively high when the basement membrane curves posteriorly and vice versa. The cells have vesicular oval or rounded basely located nuclei, which contain a distinct nucleolus. In H\&E stain their cytoplasm is lightly acidophilic (Fig A). The polyhedral cells are arranged into 7-8 layers having oval or rounded darkly stained nuclei and deeply stained acidophilic cytoplasm. The squamous cells are represented by 5-6 layers of flattened cells with darkly stained nuclei. With H\&E the cytoplasm is stained lightly eosinophilic in the deep cells but it is more deeply stained in the superficial ones (Fig A). The next membrane encountered is the external limiting membrane or membrane of Bowman which is prominent in camel cornea. It is more intimately attached to the substantiapropria and is considered to be a part of that portion of the cornea. This membrane, stains positively with PAS (Fig. C) because it is rich in mucopolysaccharides. Underlying the epithelium is a thick substantiapropria that accounts for more than $90 \%$ of the thickness of the cornea. The substantiapropria itself is composed of collagenous fibers which are stained light green with the Trichrom staining (Fig. B), the fibers are arranged in regular layers parallel to each other. Like the corneal epithelium, the substantiapropria is avascular.

The predominating cell type of the corneal substantiapropria is the fibroblast that located mainly between the collagen layers. These cells are elongated and branched, with comparatively little cytoplasm. The internal limiting membrane or Descemet's membrane is a fairly thick glossy, homogeneous membrane, reacts positively with PAS (Fig C) indicating that this membrane is rich in mucopolysaccharides. It consists mainly of collagen fibers which appear light green with Trichrom staining (Fig B). In H\&E-stained sections, the posterior limiting lamina appears as a highly refractile, thick amorphous layer. The inner most or posterior layer of the cornea is corneal endothelium. It consists of single layer of low cuboidal cells or single layer of flattened cells with the nuclei lying parallel to the internal limiting membrane, which is in direct contact with the aqueous humor of the anterior chamber. It is separated from the substantiapropria by the narrow, refractile Descemet's membrane.

\section{Immunohistochemistry}

The immuno-staining techniques used in this study allowed us to determine the distribution of the connexin 43 within cornea of one humped camel. The corneal epithelium showed positive staining reaction for connexin 43 evident mainly at the basal cell layer and polyhedral cell layers, while it showed negative reaction at the squamous cell layer. The immunostaining reaction of the corneal epithelial cells was higher in the basal cell layer than in the polyhedral cell layers. As Fig D and E shows, the expression of CX43 in corneal epithelial layer in basal cell layer as punctate staining pattern along the lateral plasma membrane between the cells and at the apical surface. However no reaction was evident in the collagen fiber of the stroma. The fibroblast cells of the corneal stroma revealed moderate immuno-staining for connexin 43 (Fig D). 


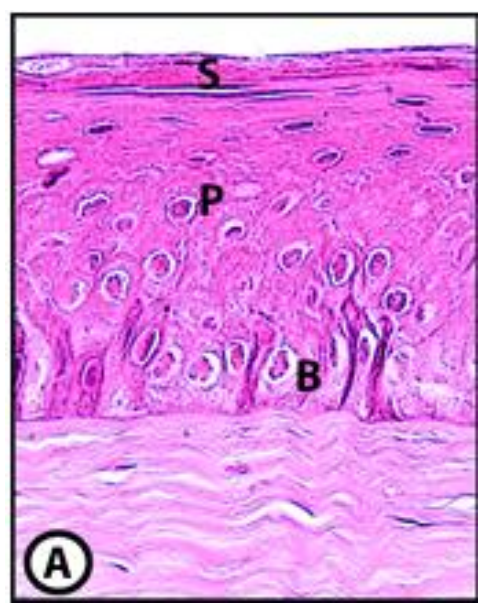

Fig. A: Staining of the camel cornea with HE (X40).

$\mathrm{B}=$ Basal cells

$\mathrm{P}=$ Polyhedral cells

$\mathrm{S}=$ Squamous cell

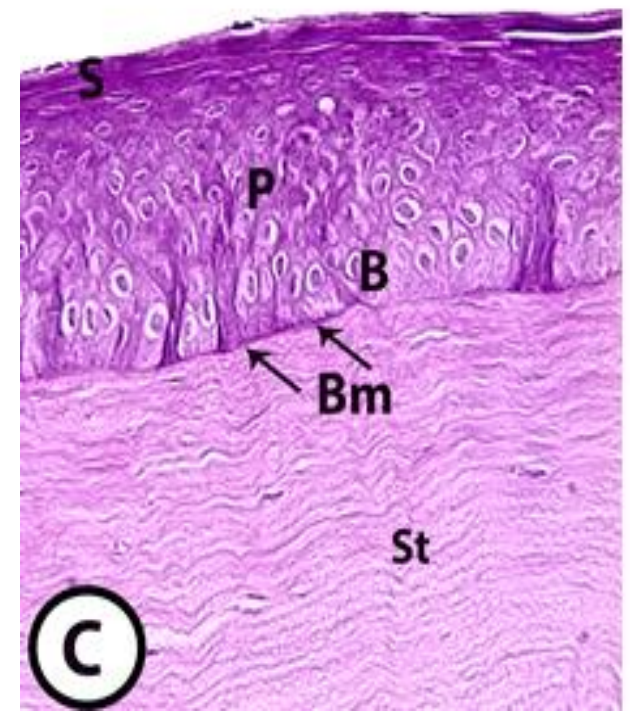

Fig. C: Staining of the camel cornea with PAS (X40).

$\mathrm{B}=$ Basal cells

$\mathrm{P}=$ Polyhedral cells

$\mathrm{S}=$ Squamous cell

$\mathrm{Bm}=$ Bowman's membrane

$\mathrm{St}=$ Substantiapropria (stroma)

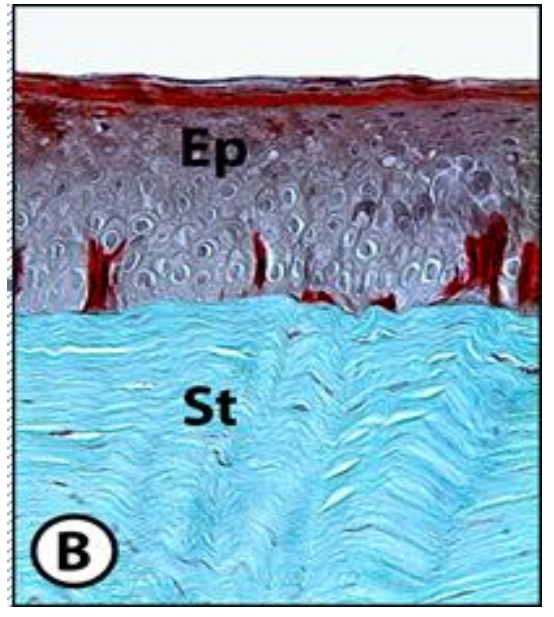

Fig. B: Staining of the camel cornea with Goldner Trichrom stain (X40).

Ep = Epithelium

St $=$ Substantiapropria (stroma)

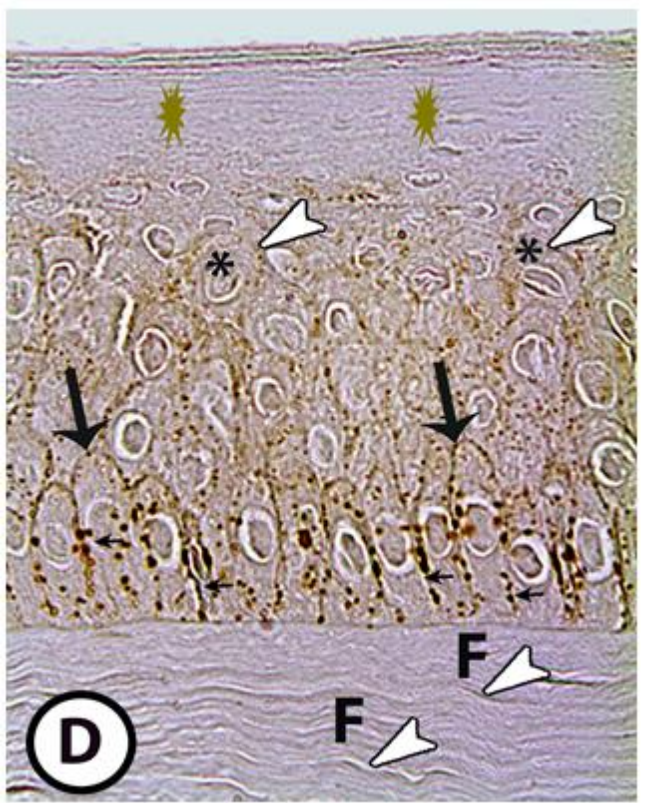

Fig. D: The immuno-staining expression of the CX 43 in corneal epithelial cells was higher in the basal cell layer than in the polyhedral cell layers $\left(^{*}\right.$, white head arrows) and negative reaction at the squamous cell layer (brown stars).

The expression of CX43 in corneal epithelial layer in basal cell layer as punctate staining pattern along the lateral plasma membrane between the cells (small black arrows) and at the apical surface (large black arrows). However no reaction was evident in the collagen fiber of the stroma. The fibroblast cells (F) of the corneal stroma revealed moderate immuno-staining for connexin 43 (white large arrow head). 


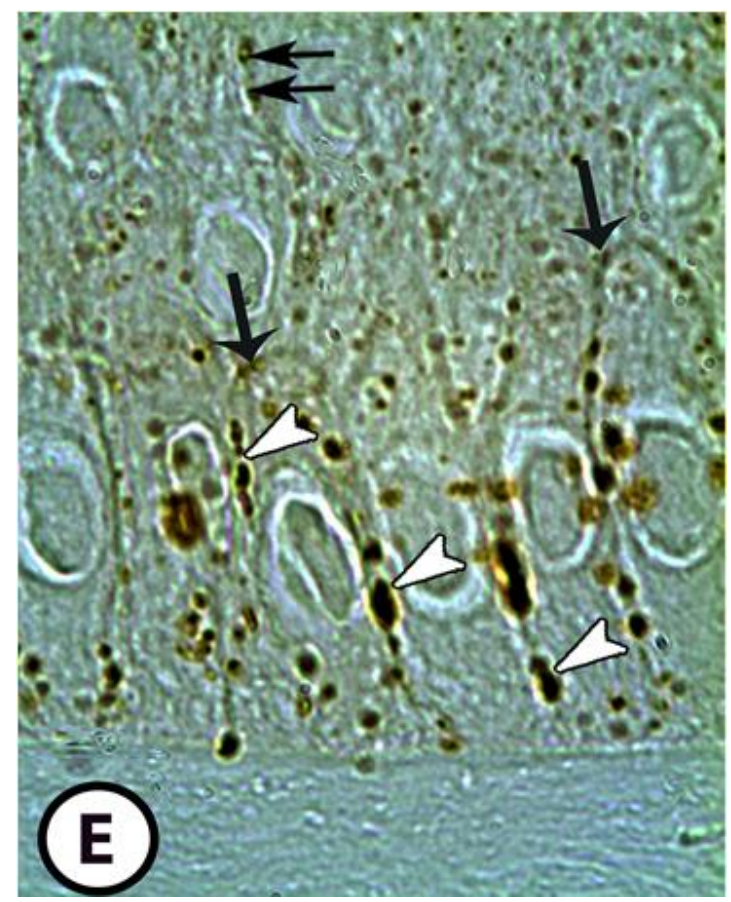

Fig. E: Higher magnification from the perviuios Image. The expression of CX43 in corneal epithelial layer in basal cell layer as punctate staining pattern along the lateral plasma membrane between the cells (white arrow heads) and at the apical surface (large black arrows). The immuno-staining expression of the CX 43 in corneal epithelial cells was moderate in the polyhedral cell layers (two black arrows).

\section{DISCUSSION}

Gap junctions are known to serve diverse functions connected to the specific needs of the tissues in which they are expressed (Kumar and Gilula, 1996 and Simon et al., 1998).

Therefore, determining the constituents of the corneal gap junction system and their specific expression patterns is essential for understanding the physiology and pathology of the corneal avascular stratified epithelium (Shurman et al., 2005). CX 43 is an important gap junction protein that contributes crucially to epithelial and stromal integrity of cornea (Gatzoufas et al., 2008). Cornel gap junctions have several important functions. It mediate the intercellular transport of ions and metabolites of low molecular weight in the basal cell layer and stroma. The transport occurred through complicated phosphorylation induced -mechanism of activation and determines the cell metabolic synchrony and corporation within the corneal epithelium as well as corneal differentiation and homoeostasis (Lampe et al., 2000 and Solan et al., 2005). In the present study we demonstrated the expression of gap junction protein CX43 in basal cell layer as punctate staining pattern along the lateral plasma membrane between the cells and at the apical surface. Similar observation was mentioned by Shurman et al. (2005) in humen corneal epithelium. They described that the CX 43 expression as characteristics punctate plasma membrane staining indicative of gap junctional plaques. The expression of CX 43 in camel was also detected with less intensity in the suprabasal polyhedral cell layer.

The expression of CX 43 in the basal cell layer was detected by several authors: in human corneal epithelium by Gatzoufas et al. (2008) and in rat corneal epithelium by Dong et al. (1994) but with different pattern of expression than camel corneal epithelium. In both species these authors detected that CX 43 was expressed continuously along the entire basal layer of basement membrane. In contrast, Shurman et al. (2005) mentioned that the $\mathrm{Cx} 43$ was localized to the apical surface in the basal layer and was found at plasma membranes throughout the basal and suprapasal layers. The observations of Shurman et al. (2005) agrees with our result.

Similar results were recorded in this work in corneal epithelium of camel agrees with results mentioned by Matic et al. (1997) and Xiaoyong Yuan et al. (2009). Matic et al. (1997) documented that the Cx43 was widely expressed throughout all layers of rodent corneal epithelium, with exception of the most superficial corneal cell, and Xiaoyong Yuan et al. (2009) were described that $\mathrm{Cx} 43$ was localized to the basal and suprabasal epithelial cells in monkey 
corneal epithelium. The Expression was detected in fibroblast in stroma layer in cornea of the camel. Similar expression was recorded by (Gatzoufas et al., 2008) in human corneal epithelium.

\section{REFERENCES}

Bancroft, J.D and Gamble, M. (2002): Theory and Practice of Histological and Histochemical Techniques. 3re Edn, Butter Worths. Cell.

Cao, F.; Eckert, R. and Elfgang, C. (1998): A quantitative analysis of connexin specific permeability differences of gap junctions expressed in HeLa transfectants and Xenopus oocytes. J Cell Sci. 111: 31-43.

Daniel, L. Shurman, Lisa Glazewski, Anna Gumpert, J. ames D. Zieske and Gabriele Richard (2005): In vivo and in vitro expression of connexin in humen corneal epithelium. Investigative Ophthalmology and Visual Science. (46): 6.

Differential expression of two gap junction proteins in corneal epithelium (1994): Eur J Cell Biol. 64(1): 95-100.

Dong $Y$, Roos M, Gruijters T, Donaldson P, Bullivant $S$, Beyer E, Kistler J.

Gatzioufas, Z.; Charalambous, P. and Thanos, S. (2008): Reduced expression of the gap junction protein connexin 43 in keratoconus. Eye. 22:294-299.

Hertzberg, EL. (2000): eds. Gap Junctions, Advances in Molecular and Cell Biology. JAI Press Greenwich, CT.

Joyce, NC.; Harris, DL. and Zieske, JD. (1998): Mitotic inhibition of corneal endothelium in neonatal rats. Invest Ophthalmol Vis Sci. 39: $2572-$

Kumar, NM. and Gilula, NB. (1996): The gap junction communication channel. Cell; 84: 381-388.

Lampe, PD.; Tenbroke, EM.; Burt, JM.; Kurata, WE.; Johnson, RG. and Lau, AF. (2000): Phosphorylation of connexin 43 on serine 368 by protein kinase $\mathrm{C}$ regulates gap junctional
communication.J Cell Biol; 149(7): 15031512.

Laux-Fenton, WT.; Donaldson, PJ.; Kistler, J. and Green, CR. (2003): Connexin expression patterns in the rat cornea: molecular evidence for communication compartments. Cornea. 22: 457-464.

Matic, M.; Petrov, IN.; Rosenfeld, T. and Wolosin, JM. (1997): Alterations in connexin expression and cell communication in healing corneal epithelium. Invest Ophthalmol Vis Sci. 38: 600-609.

Mohay, J. and McLaughlin, BJ. (1995): Corneal endothelial wound repair in normal and mitotically inhibited cultures. Graefes Arch Clin Exp Ophthalmol. 233: 727-736.

Saez, JC.; Berthoud, VM.; Branes, MC.; Martinez, $A D$. and Beyer, EC. (2003): Plasma membrane channels formed by connexins: their regulation and functions. Physiol Rev. 83: 1359-1400.

Simon, AM.; Goodenough, DA. and Paul, DL. (1998): Mice lacking Connexin 40. Have cardiac conduction abnormalities characteristic of atrioventricular block and bundle branch block. Curr Biol. 8: 295-258.

Solan, JL. and Lampe, PD. (2005): Connexin phosphorylation as a regulatory event linked to gap junction channel assembly. Biochim Biophys Acta. 1711 (2): 154-163.

Williams, K. and Watsky, M. (2002): Gap junctional communication in the human corneal endothelium and epithelium. Curr Eye Res. 25: 29-36.

Williams, KK. and Watsky, MA. (2004): Bicarbonate promotes dye coupling in the epithelium and endothelium of the rabbit cornea. Curr Eye Res. 28: 109-120.

Xiaoyong Yuan, MD, Zhuo Chen, MD, Zhuo Yang, BS, Junfang Gao, Aijun Zhang, MS, Samuel M. Wu, and Roy Jacoby (2009): Expression Pattern of Connexins in the Corneal and Limbal Epithelium of a Primate. Cornea; 28 (2).

Yeager, M. and Nicholson, BJ. (1996): Structure of gap junction intercellular channels. Curr Opin Struct Biol; 6: 183-192.

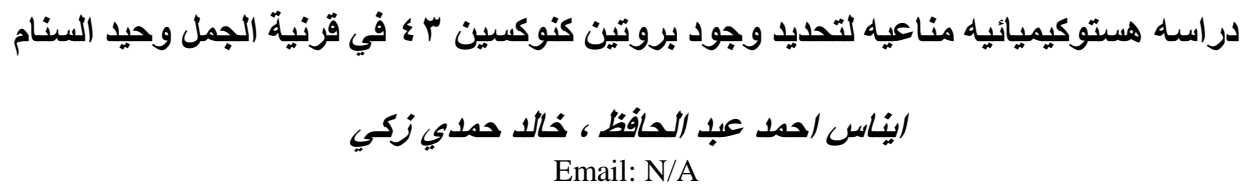

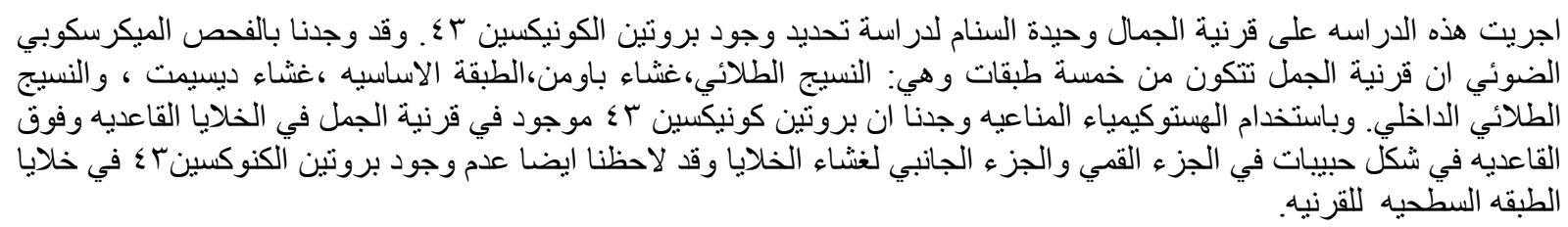

